\title{
Gas accretion from the cosmic web feeding disk galaxies
}

\author{
J. Sánchez Almeida ${ }^{1,2}$, A. Olmo-García ${ }^{1,2}$, B. G. Elmegreen ${ }^{3}$, \\ C. Muñoz-Tuñón ${ }^{1,2}$, D. M. Elmegreen ${ }^{4}$, M. E. Filho ${ }^{1,2,5,6,7}$, \\ E. Pérez-Montero ${ }^{8}$ and R. Amorín ${ }^{9}$ \\ ${ }^{1}$ Instituto Astrofísica de Canarias, 38200 La Laguna, Tenerife, Spain \\ ${ }^{2}$ Departamento de Astrofísica, Universidad de La Laguna, Spain \\ ${ }^{3}$ IBM Research Division, T.J. Watson Research Center, Yorktown Heights, NY 10598, USA \\ ${ }^{4}$ Department of Physics and Astronomy, Vassar College, Poughkeepsie, NY 12604, USA \\ ${ }^{5}$ SIM/CENTRA, Lisbon, Portugal \\ ${ }^{6}$ Centro de Astrofísica da Universidade do Porto, Porto, Portugal \\ ${ }^{7}$ Instituto de Astrofísica e Ciências do Espaço, Universidade de Lisboa, Lisboa, Portugal \\ ${ }^{8}$ Instituto de Astrofísica de Andalucía, CSIC, Granada, Spain \\ ${ }^{9}$ INAF-Osservatorio Astronomico di Roma, Monte Porzio Catone, Italy
}

\begin{abstract}
Disk galaxies in cosmological numerical simulations grow by accreting gas from the cosmic web. This gas reaches the external disk, and then spirals in dragged along by tidal forces and/or disk instabilities. The importance of gas infall is as clear from numerical simulations as it is obscure to observations. Extremely metal poor (XMP) galaxies seem to be the best example we have of the gas accretion process at work. They have large off-center starbursts which show significant metallicity drop compared with the host galaxy. This observation is naturally explained as a gas accretion event caught in the act. We present preliminary results of the kinematical properties of the metal poor starbursts in XMPs, which suggest that the starbursts are kinematically decoupled entities within the host galaxy.
\end{abstract}

Keywords. galaxies: abundances, galaxies: formation, intergalactic medium, galaxies: irregular, galaxies: kinematics and dynamics, galaxies: starburst

\section{Introduction}

Galaxies with a metallicity smaller than a tenth of the solar metallicity are known as extremely metal poor (XMP). Since metals are primarily produced by stars, XMPs are chemically primitive. They also show signs of being structurally primitive, as if XMPs were disks in early stages of assembly, with bulky off-center starbursts giving them a characteristic cometary shape (e.g., Morales-Luis et al. 2011, Elmegreen et al. 2016), and with massive HI envelopes (e.g., Filho et al. 2103). Reinforcing such primitive character, their chemical composition is often non-uniform with the low metallicity mostly in the regions of intense star formation. The existence of chemical inhomogeneities is particularly revealing because the timescale for mixing in disk galaxies is short, of the order of a fraction of the rotational period (e.g., Yang \& Krumholz 2012). It implies that the metal-poor gas in XMPs was recently accreted from a nearly pristine gas cloud, very much in the line of the expected cosmic cold-flow accretion (e.g., Dekel et al. 2009), that has been so difficult to observe directly (e.g., Sánchez Almeida et al. 2014). Thus, XMPs seem to be local galaxies that are growing through the physical processes that shaped the disks of more massive galaxies in the early universe.

The presence of chemical inhomogeneities in most XMPs is the touchstone of the whole scenario, since it unequivocally shows the star formation to be feeding from external metal-poor gas. In order to put on a firm observational basis the presence of these 


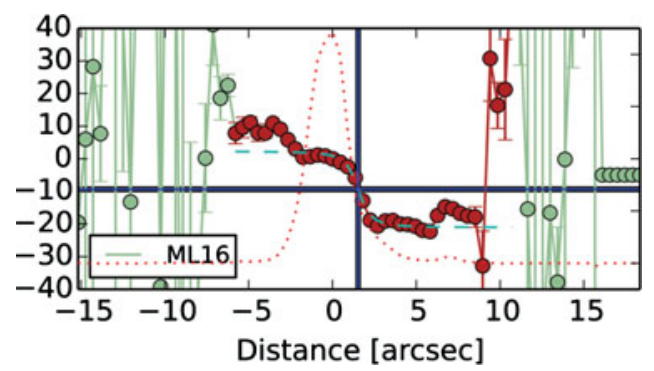

Figure 1. Rotation curve along the major axis of one of the XMPs, ML16 (J1145+50). The points with error bars represent observations, and they are shown in red when the velocity estimate is reliable. Distances along the slit are given in arcsec whereas velocities (in the ordinate axis, without label) are in $\mathrm{km} \mathrm{s}^{-1}$. The dotted line shows the variation of the $\mathrm{H} \alpha$ flux along the slit. The peak $\mathrm{H} \alpha$ emission, at distance zero, marks the position of the starburst. It does not coincide with the center of rotation (vertical solid line). The dashed line represents a fit of the observed velocity to the universal rotation curve by Salucci et al. (2007).

inhomogeneities, we measured the oxygen abundance along the major axes of ten XMPs (Sánchez Almeida et al. 2015), using a variant of the direct method (Pérez-Montero 2014). In nine out of the ten cases, sharp metallicity decrements were found, leaving little doubt on the reality and ubiquity of the drops. In order to complement these measurements, we carried out a new set of observations to characterize the kinematical and dynamical properties of the XMPs. The original spectra used to infer metallicities do not possess enough spectral resolution to carry out kinematic studies. Here we give preliminary results of the analysis of this new data set, which suggest that the starbursts are kinematically decoupled from the host galaxy, hinting at the external origin of the galaxy gas.

\section{Kinematical properties of XMPs}

$\mathrm{H} \alpha$ long-slit spectra of the XMPs described in the previous section were taken with the ISIS spectrograph at the $4.2 \mathrm{~m}$ WHT on La Palma, which provides a spectral resolution around 7000. Integrating two hours on source, the resulting spectra allow us to compute line centers and line widths with a precision of a few $\mathrm{km} \mathrm{s}^{-1}$.

The observed XMPs tend to rotate, with typical rotation velocities of $20-30 \mathrm{~km} \mathrm{~s}^{-1}$, velocity dispersions as large as the rotation, and rotation curves showing significant deviations from smoothness. The center of rotation does not necessarily coincide with the position of the starburst. ML16 (J1145+50) is shown as example in Fig. 1.

We also find a curious systematic for the variation of the velocity and the velocity dispersion across each starbursts. It is summarized in Fig. 2, which shows the variation of the velocity and the velocity dispersion within the starburst for all starbursts. The two main observational findings are: (1) the bulk velocity turns out to be constant within the starburst, and (2) the velocity dispersion follows two distinct patterns that we denote as Type 0 (Fig. 2, top panel) and Type 1 (Fig. 2, bottom panel). In Type 0, the velocity dispersion presents a local maximum at the center of the starburst. In Type 1, however, the dispersion increases across the starburst toward the position that is closest to the dynamical center of the host galaxy.

So far we can only speculate on how to interpret these two results. As for number (1), it indicates that the starburst is a separate entity within the galaxy since it has its own rotational velocity. As for (2), the excess of velocity dispersion in the center-side of the Type 1 starbursts may indicate an intensification of the turbulence of the gas in that particular part of the starburst. Such increase may indicate the collision of the starburst 

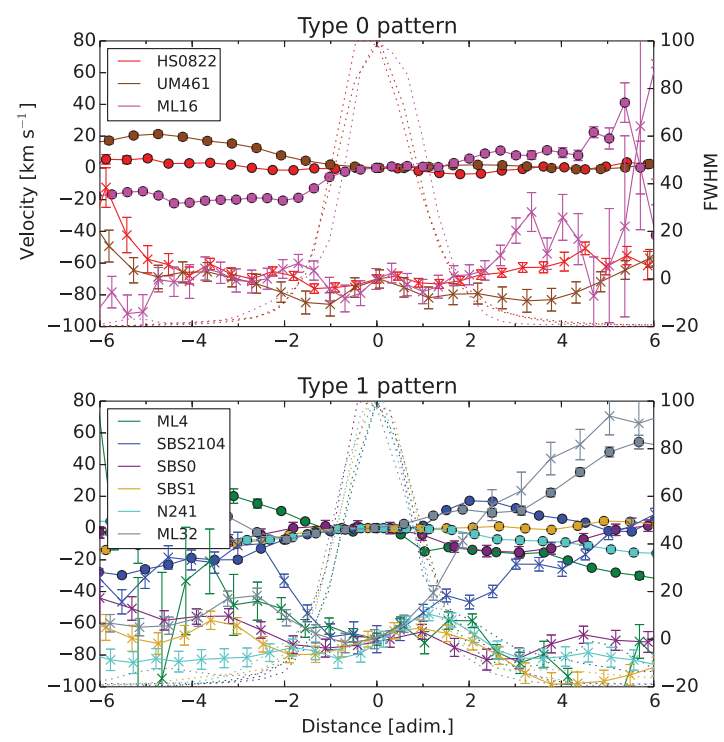

Figure 2. Variation of the velocity (bullet symbols) and velocity dispersions (asterisks) across the starbursts of the XMPs. Distances, in the abscissa axis, are referenced to the position of the starbursts and normalized to its size. For this reason the variation of the $\mathrm{H} \alpha$ flux across the starbursts, shown as dotted lines, is always centered at distance zero and has always the same width. The velocity turns out to be constant within the starburst, whereas the velocity dispersion follows two distinct patterns, labelled as Type 0 (shown in the top panel) and Type 1 (bottom panel). In Type 0, the dispersion presents a local maximum at the center of the starburst. In Type 1, however, the dispersion increases across the starburst toward the position that is closest to the galaxy dynamical center. Different colors refer to different galaxies as indicated by the insets. The nicknames used for the XMPs are ML4 $\equiv J 0303-01, H S 0822 \equiv J 0825+35, S B S 0 \equiv J 0944:+54, \mathrm{SBS} 1 \equiv \mathrm{J} 1132+57, \mathrm{ML} 16 \equiv \mathrm{J} 1145+50$, $\mathrm{UM} 461 \equiv \mathrm{J} 1151-02, \mathrm{SBS} 2104 \equiv \mathrm{J} 2104-00, \mathrm{ML} 32 \equiv \mathrm{J} 2302+00, \mathrm{~N} 214 \equiv \mathrm{J} 1444+42$.

gas with the interstellar medium of the host galaxy, as if the clumps were inspiring toward the galaxy center. This migration to the galaxy center is expected from tidal forces acting upon massive gas clumps; see, e.g., Elmegreen et al. (2008), Ceverino et al. (2016). The kinematical properties that we find suggest that the starbursts are independent entities within the host galaxy.

These observational results and the subsequent interpretation are the subject a work in preparation by Olmo-García et al. (2016).

\section{References}

Ceverino, D., Sánchez Almeida, J., Muñoz Tuñón, C., et al. 2016, MNRAS, 457, 2605

Dekel, A., Birnboim, Y., Engel, G., et al. 2009, Nature, 457, 451

Elmegreen, B. G., Bournaud, F., \& Elmegreen, D. M. 2008, ApJ, 688, 67

Elmegreen, D. M., Elmegreen, B. G., Sánchez Almeida, J., et al. 2016, ApJ, submitted

Filho, M. E., Winkel, B., Sánchez Almeida, J., et al. 2013, A\& A, 558, A18

Morales-Luis, A. B., Sánchez Almeida, J., Aguerri, J. A. L., et al. 2011, ApJ, 743, 77

Pérez-Montero, E. 2014, MNRAS, 441, 2663

Salucci, P., Lapi, A., Tonini, C., et al. 2007, MNRAS, 378, 41

Sánchez Almeida, J., Elmegreen, B. G., Muñoz-Tuñón, C., et al. 2014, A\&̛ARv, 22, 71

Sánchez Almeida, J., Elmegreen, B. G., Muñoz-Tuñón, C., et al. 2015, ApJL, 810, L15

Yang, C.-C. \& Krumholz, M. 2012, ApJ, 758, 48 